\title{
Vitamin D supplementation during pregnancy: state of the evidence from a systematic review of randomised trials
}

\author{
Daniel E Roth, ${ }^{1,2,3}$ Michael Leung, ${ }^{2}$ Elnathan Mesfin, ${ }^{2}$ Huma Qamar, ${ }^{2,3}$ Jessica Watterworth, ${ }^{2,3}$ \\ Eszter Papp ${ }^{2}$
}

${ }^{1}$ Department of Paediatrics, Hospital for Sick Children and University of Toronto, Toronto, ON, Canada

${ }^{2}$ Centre for Global Child Health and SickKids Research Institute, Hospital for Sick Children, Toronto, ON, Canada

${ }^{3}$ Department of Nutritional Sciences, Faculty of Medicine, University of Toronto, Toronto, ON, Canada

Correspondence to: D E Roth daniel.roth@sickkids.ca

Additional material is published online only. To view please visit the journal online.

Cite this as: BMJ 2017;359:j5237 http://dx.doi.org/10.1136/bmj.j5237

Accepted: 22 October 2017

\section{ABSTRACT}

OBJECTIVES

To estimate the effects of vitamin D supplementation during pregnancy on 11 maternal and 27 neonatal/ infant outcomes; to determine frequencies at which trial outcome data were missing, unreported, or inconsistently reported; and to project the potential contributions of registered ongoing or planned trials.

\section{DESIGN}

Systematic review and meta-analysis of randomised controlled trials; systematic review of registered but unpublished trials.

\section{DATA SOURCES}

Medline, Embase, PubMed, Cochrane Database of Systematic Reviews, and Cochrane Central Register of Controlled Trials from inception to September 2017; manual searches of reference lists of systematic reviews identified in the electronic search; and online trial registries for unpublished, ongoing, or planned trials.

ELIGIBILITY CRITERIA FOR STUDY SELECTION

Trials of prenatal vitamin D supplementation with randomised allocation and control groups administered placebo, no vitamin $D$, or vitamin $D$ $\leq 600 \mathrm{IU} /$ day (or its equivalent), and published in a peer reviewed journal.

\section{WHAT IS ALREADY KNOWN ON THIS TOPIC}

Numerous randomised trials and systematic reviews of vitamin $D$

supplementation during pregnancy have been published, with conflicting results and conclusions

Recommendations regarding vitamin D supplementation vary widely among medical and professional organisations, and WHO currently recommends against routine prenatal vitamin D supplementation

\section{WHAT THIS STUDY ADDS}

Systematic review and meta-analyses of 43 trials including 8406 participants showed that prenatal vitamin D supplementation was associated with increased maternal and cord serum 25-hydroxyvitamin D concentrations, increased mean birth weight, reduced the risk of small for gestational age, reduced the risk of wheeze in offspring, and increased infant length at one year of age

There was a lack of evidence of benefits of prenatal vitamin $D$ supplementation for maternal health conditions related to pregnancy, no effect on other birth outcomes of public health importance such as preterm birth, and scant evidence on safety outcomes

Few of the trials were designed to test the effect of vitamin D on clinical or functional outcomes, and most trials were small and at overall high or uncertain risk of bias

Thirty five planned or ongoing prenatal vitamin D trials could contribute an additional 12530 participants to future systematic reviews

\section{RESULTS}

43 trials (8406 participants) were eligible for meta-analyses. Median sample size was 133 participants. Vitamin D increased maternal/cord serum concentration of 25 -hydroxyvitamin $D$, but the dose-response effect was weak. Maternal clinical outcomes were rarely ascertained or reported, but available data did not provide evidence of benefits. Overall, vitamin D increased mean birth weight of $58.33 \mathrm{~g}$ (95\% confidence interval $18.88 \mathrm{~g}$ to 97.78 g; 37 comparisons) and reduced the risk of small for gestational age births (risk ratio $0.60,95 \%$ confidence interval 0.40 to 0.90 ; seven comparisons), but findings were not robust in sensitivity and subgroup analyses. There was no effect on preterm birth $(1.0,0.77$ to 1.30; 15 comparisons). There was strong evidence that prenatal vitamin $D$ reduced the risk of offspring wheeze by age 3 years $(0.81,0.67$ to 0.98 ; two comparisons). For most outcomes, meta-analyses included data from a minority of trials. Only eight of 43 trials (19\%) had an overall low risk of bias. Thirty five planned/ongoing randomised controlled trials could contribute 12530 additional participants to future reviews.

\section{CONCLUSIONS}

Most trials on prenatal vitamin D published by September 2017 were small and of low quality. The evidence to date seems insufficient to guide clinical or policy recommendations. Future trials should be designed and powered to examine clinical endpoints, including maternal conditions related to pregnancy (such as pre-eclampsia), infant growth, and respiratory outcomes.

\section{SYSTEMATIC REVIEW REGISTRATION}

PROSPERO CRD42016051292

\section{Introduction}

Vitamin D continues to garner substantial attention from clinicians, researchers, and the public. Beyond its established influence on bone growth and calcium homeostasis, ${ }^{1}$ vitamin D and its related metabolites have hypothesised effects on the risk of cardiovascular diseases, ${ }^{2}$ cancer, $^{3}$ respiratory infections, ${ }^{4}$ asthma, ${ }^{5}$ conditions related to pregnancy (such as pre-eclampsia, gestational diabetes), and birth outcomes. ${ }^{6}$ Concern that vitamin D deficiency is a global public health problem has been sparked by numerous reports of the high proportion of individuals in studies throughout the world who have relatively low serum/plasma concentrations of 25-hydroxyvitamin D $(25(\mathrm{OH}) \mathrm{D}){ }^{7}$ the conventional circulating biomarker of vitamin D status. ${ }^{8}$ There has been particular attention to the high prevalence of vitamin D deficiency in pregnant women and newborns. ${ }^{7}$

In 2010, dietary reference intakes for vitamin D for Canada and the US from the Institute of Medicine were 
based solely on bone health-particularly in young children (for instance, rickets) and older adults (for instance, osteoporosis). ${ }^{1}$ Considerable skepticism was cast onto the link between vitamin $\mathrm{D}$ and extraskeletal health conditions or the potential benefits (versus risks) of intakes larger than the recommended dietary allowance (600 IU/day for most children and adults). ${ }^{1}$ The institute's report did not recommend higher intakes during pregnancy and lactation. ${ }^{1}$ A 2016 update of the Cochrane Collaboration systematic review and metaanalysis of trials of vitamin D in pregnancy (versus placebo or no supplement), however, tentatively concluded that prenatal vitamin D supplementation "may reduce the risk of pre-eclampsia, low birthweight and preterm birth." ${ }^{6}$ Other recent systematic reviews that included trials with active control arms (in which participants received vitamin D) acknowledged the potential effect of vitamin D on fetal growth ${ }^{910}$ but did not find evidence of effects on other maternal, fetal, or infant outcomes. ${ }^{9-11}$ Citing the 2016 Cochrane review, ${ }^{6}$ the World Health Organization guidelines for antenatal care advise against routine vitamin D supplementation in pregnancy. $^{12}$ Dietary and supplementation recommendations, however, vary widely across other professional organisations (appendix 1), and there remains a lack of consensus on target health outcomes, indications for prenatal supplementation, or evidence based regimens for supplement dose (or fortification strategies).

Given the numerous trials on prenatal vitamin D published since 2015 and the restricted scope of the 2016 Cochrane review, we undertook an updated systematic review of vitamin D in pregnancy to assess the current and future state of the evidence from randomised controlled trials. We specifically aimed to estimate the effects of vitamin D supplementation during pregnancy on maternal, neonatal, and infant outcomes, taking into consideration the quality of available evidence and heterogeneity across studies; determine frequencies at which relevant outcome variable data were missing, unreported, or inconsistently reported among trials that were otherwise eligible for inclusion; and identify ongoing or planned studies of vitamin D supplementation during pregnancy to project the state of evidence that could accrue in the next decade.

\section{Methods}

Literature search strategy

The protocol was registered (CRD42016051292) and conducted following PRISMA (Preferred Reporting Items for Systematic Reviews and Meta-Analyses) guidelines. ${ }^{13}$ We sought relevant articles reporting completed studies by searching electronic databases including Medline, Medline in process, Embase, PubMed, the Cochrane Database of Systematic reviews, and the Cochrane Central Register of Controlled Trials (appendix 2) and manual searches of reference lists of any systematic reviews identified in the previous step. Searches were initially done in July 2016 and most recently updated in September 2017.

\section{Eligibility criteria}

We included trials of prenatal vitamin D supplementation in which

- Supplementation was vitamin D given alone or in combination with a cointervention that was similar across multiple arms

- Participants were pregnant at enrolment or enrolled before pregnancy and then followed-up in pregnancy

- Allocation into the parallel intervention groups at or after enrolment was randomised

- At least one trial group received placebo, no vitamin $\mathrm{D}$, or up to $600 \mathrm{IU} /$ day (or a less frequent dose that would be about equivalent to $600 \mathrm{IU} /$ day-for example, $4200 \mathrm{IU} /$ week) during pregnancy

- At least one of the articles describing the trial was a full text report published in a scientific journal or in another peer reviewed format.

Vitamin D could be given in either form (vitamin D2 or D3), administered at any dose, by any route (oral or intramuscular), and at any frequency-“regular" dosing in which supplementation was offered at least three times in a regular/recurrent manner (such as daily, weekly, monthly) and "bolus" dose regimens in which the supplement was administered only once or twice. Outcome data were not considered in determination of eligibility. There were no language restrictions at the screening stage, but all eligible studies were reported in English.

Trials were eligible for inclusion in meta-analysis if all five inclusion criteria were met. Studies that met only the first two inclusion criteria were ineligible for inclusion in meta-analysis but were reviewed separately (that is, trials that had parallel but nonrandomised intervention group assignment, trials with control arms administered vitamin D dose >600 IU/day, or those whose results were not reported in a full text publication). We excluded from the systematic review single arm vitamin D trials (such as pharmacokinetic studies); trials in which the intervention was administered only before conception or after delivery; observational cohorts rather than parallel group trials (that is, intervention group assignment was based on review of medical records or participant recall or there was a lack of sufficient evidence in the published report to indicate that participants were assigned prospectively to intervention groups); and trials in which differences in cointervention(s) across trial arms prevented inferences specifically about vitamin D. Two independent reviewers screened abstracts and full text articles for inclusion using a web based systematic review platform (Covidence). Any disagreements at either screening stage were resolved through consensus of at least two reviewers.

\section{Data collection}

Two reviewers independently extracted information from eligible studies related to country, baseline characteristics of the study population including 25(OH)D summary data at enrolment, number of participantsenrolled, thenature of thesupplementation in the intervention and control arms (that is, type of 
supplementation, dosing regimen, cointerventions, duration), and biochemical and clinical outcomes (appendix 3). For continuous outcomes, we extracted means and corresponding standard deviations in each arm; if trials reported other statistics (such as median and interquartile range), we converted estimates to means and SDs using conventional approaches. ${ }^{14}$ For binary outcomes, we extracted the number of participants who experienced the event (numerator) and total number of individuals at risk of an observed event (denominator), thereby excluding participants lost to follow-up from the denominator. Unless otherwise specified by the original authors, the number of newborns at risk of any birth related outcome measure (such as preterm birth) was limited to liveborn infants.

If available, we used multiple published articles from the same trial population to extract outcome data. We contacted principal investigators or corresponding authors of eligible studies after 1999 for data on outcomes if an outcome was mentioned in published reports but the data were not reported; results for a specified outcome were presented only in qualitative terms (such as "not significant"); or reported data relevant to one of the specified outcomes were labeled or presented with summary measures that could not be incorporated into the meta-analysis.

\section{Assessment of risk of bias}

Two reviewers used the Cochrane Collaboration tool for assessing risk of bias to independently assign quality scores to the trials. ${ }^{15}$ Each study was assessed as having low, high, or unclear risk of bias on seven criteria: random sequence generation, allocation concealment, blinding of participants and personnel, blinding of outcome assessment, incomplete outcome data, selective reporting, and other sources of bias. Any discrepancies between reviewers were resolved through consensus.

\section{Outcome definitions}

To enable coherent interpretations of primary metaanalyses related to outcomes that rely on clinical diagnosis or results of diagnostic tests, we specified minimum criteria for case definitions and methods of ascertainment (for instance, routine data collection procedures that were reportedly used to ascribe diagnoses to individual trial participants) (appendix 3). For example, for pre-eclampsia we required evidence that participants were scheduled to undergo routine blood pressure monitoring and urinary protein testing (the latter being undertaken at least among women with hypertension). For gestational diabetes, we required routine glucose challenge testing, oral glucose tolerance test, and/or measurement of $\mathrm{HbA}_{1 \mathrm{c}}$ during follow-up. For biochemical safety events (such as hypercalcaemia), we required that investigators reported a schedule of routine testing of biomarkers in blood/urine. We relaxed case definitions/methods of ascertainment criteria in sensitivity analyses in which we included any trials that mentioned the outcome irrespective of case definitions or methods of ascertainment.

\section{Statistical methods}

We performed standard meta-analyses to generate a pooled risk ratio and 95\% confidence interval for each dichotomous outcome or a weighted mean difference and 95\% confidence interval for each continuous outcome. Random effects models with inverse variance weights were used to account for expected heterogeneity of the effect estimates. Statistical heterogeneity was quantified with the $\mathrm{I}^{2}$ statistic. ${ }^{16}$ If a trial had multiple intervention arms, they were disaggregated and included in the metaanalysis as separate intervention-control comparisons. When multiple comparisons from the same trial were included in the same meta-analysis, we divided the control group sample size corresponding to each comparison by the number of intervention arms to avoid double counting participants. ${ }^{14}$

We conducted subgroup meta-analyses according to a priori defined sources of potential clinical and methodological heterogeneity ${ }^{17}$ : control arm type (placebo versus vitamin D containing active control); dosing frequency (regular versus bolus); baseline vitamin D status categorised as low (mean $25(\mathrm{OH})$ $\mathrm{D}<30 \mathrm{nmol} / \mathrm{L}$ ) versus sufficient ( $\geq 30 \mathrm{nmol} / \mathrm{L})$, based on maternal mean $25(\mathrm{OH}) \mathrm{D}$ in the control group at enrolment ("initial baseline") or at/near delivery ("modified baseline," which theoretically takes into account the effect of active control supplementation); intervention dose (among regular dose trials), defined as low ( $<2000 \mathrm{IU} /$ day) versus high ( $\geq 2000 \mathrm{IU} /$ day). To facilitate comparisons, regular doses given less frequently than daily were converted to an equivalent daily dose (for example, $28000 \mathrm{IU} /$ week $=4000 \mathrm{IU} /$ day) and expressed as an "effective dose" (that is, intervention dose minus the vitamin D dose in the control group in the same trial). Additional covariates included type of vitamin D supplementation (D2 versus D3); geographical region based on WHO classification (Africa, Americas, South-East Asia, Europe, Eastern Mediterranean, and Western Pacific); and timing of initiation of supplementation (such as first, second, or third trimester) among regular dose trials in which supplementation continued until delivery. For outcomes that included at least 10 eligible intervention arms, we performed meta-regression using the following covariates as continuous independent variables: effective dose, change in maternal $25(\mathrm{OH}) \mathrm{D}$ (that is, difference between mean 25(OH)D in intervention and control at/near delivery), initial baseline vitamin D status, and modified baseline status.

We conducted sensitivity meta-analyses restricted to trials with recent publication (2000 or later); overall low risk of bias (low risk of bias in all seven criteria); and enrolment of generally healthy women (rather than those with a specific clinical diagnosis). To incorporate trials with zero events in both intervention and control arms (which are automatically dropped from analyses of pooled relative risks), we also did sensitivity analyses for dichotomous outcomes in which we added a "continuity correction" of 0.5 to zero cells. ${ }^{18}$ Publication bias was assessed by visual 
inspection of funnel plots for outcomes with at least 10 intervention arms. ${ }^{19}$ All analyses were conducted with Stata version 13 (College Station, TX).

\section{Missing outcome analysis}

As we could not determine the reasons for missing individual level outcome data across multiple trials, we based all primary meta-analyses on a "complete case analysis" (that is, excluding participants for whom outcomes were missing), ${ }^{20}$ which implied a missing at random assumption (that is, reasons for missing data were unassociated with the outcome). We considered data imputation methods, ${ }^{20} 21$ but available approaches require untenable or unverifiable assumptions and serve only to explore the potential change in pooled effect sizes given a departure from missing at random rather than provide more reliable estimates of effects. Therefore, we chose to contextualise the primary findings in a critical assessment of missingness for each outcome by calculating the number/proportion of all participants enrolled in each trial for whom the outcome was missing because the outcome was not reportedly assessed in any participants during the trial; unreported because of loss of follow-up or incomplete reporting of planned trial outcomes; or reported by authors but in a manner that prevented inclusion of the data in the metaanalysis. Missingness analyses were based only on the trials eligible for inclusion in meta-analyses.

\section{Identification and summary of registered ongoing/ planned trials}

We searched seven clinical trial registries (WHO, Clinicaltrials.gov, ISRCTN, ANZCTR, DRKS, EU-CTR, IRCT) to identify ongoing or planned clinical trials worldwide (appendix 4). Studies were considered eligible if they had not published results; had enrolled participants no earlier than 2009; and were considered likely to meet the criteria for inclusion in meta-analyses based on available information. We excluded duplicate trials within and between registries (identified by trial ID, title, investigators, sample sizes). We screened titles and registry pages for eligibility and verified that results of eligible trials had not been published in peer reviewed journals before September 2017. All screening stages were done in duplicate by two independent reviewers, and discrepancies were resolved through discussion among two or more reviewers.

From registry entries of eligible ongoing/planned trials, we extracted information (when available) on geographical location, study status (for example, completed but unpublished, ongoing, not yet started, status unknown), number of participants enrolled, recruitment start date, inclusion and exclusion criteria, details on vitamin D supplementation dose, frequency and duration, cointerventions, and primary and secondary outcomes that investigators listed in the registry. Studies were classified as being likely to contribute published results by 2020 (that is, studies for which at least enrolment has been completed) or 2024 (studies for which enrolment is ongoing, not yet started, or for which the status is unknown). We assessed the potential contribution of eligible planned/ ongoing prenatal vitamin D trials by identifying the number of participants and trials for the same prespecified list of maternal or child outcomes as in the systematic review of completed trials.

\section{Patient and public involvement}

No patients were involved in setting the research question or the outcome measures, nor were they involved in developing plans for design or implementation of the study. No patients were asked to advise on interpretation or writing up of results. There are no plans to disseminate the results of the research to study participants or the relevant patient community. It was not evaluated whether the studies included in the review had any patient involvement.

\section{Results}

Literature search results and study selection

Among 3810 articles identified from the literature search, 43 prenatal vitamin D trials were eligible for inclusion in meta-analyses based on data in 77 publications (fig 1; table A in appendix 5). We received responses from 10 of 16 (63\%) authors of studies we contacted for further data on outcomes that were either missing (but presumed to have been collected based

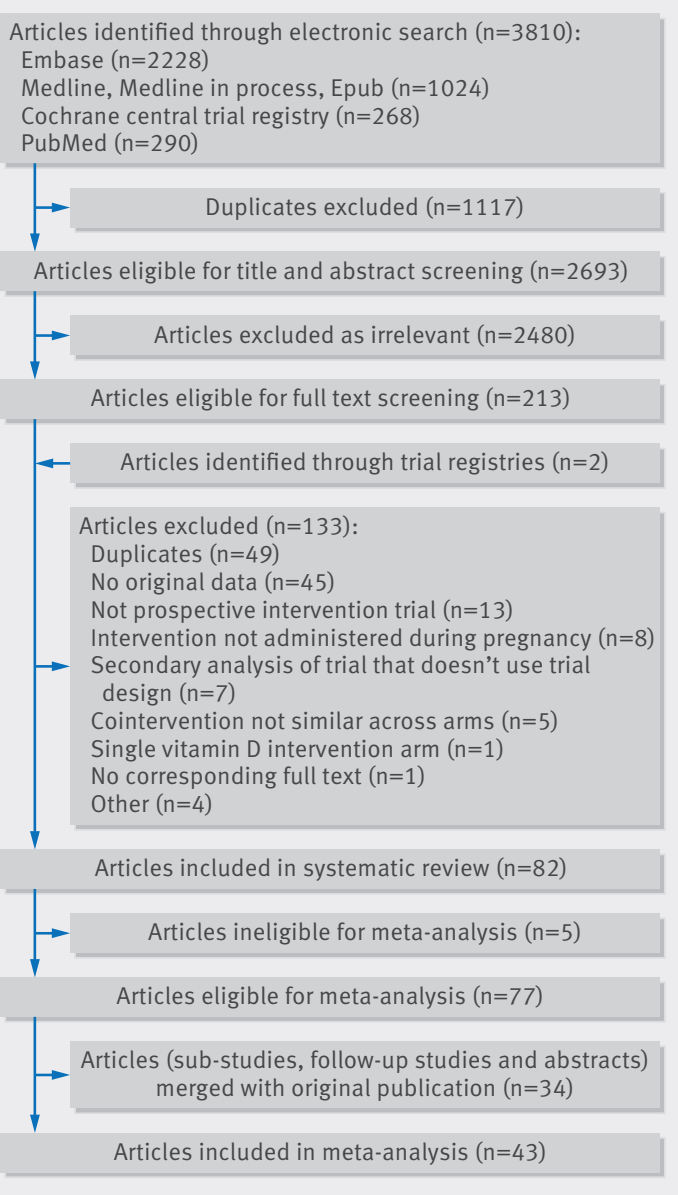

Fig 1 | Screening and selection of studies for systematic review and meta-analysis of vitamin $D$ supplementation during pregnancy 


\begin{tabular}{|c|c|c|c|}
\hline Characteristic & All trials & Regular dose trials* & $\begin{array}{l}\text { Bolus dose } \\
\text { trials* }\end{array}$ \\
\hline Trials & 43 & $37 \dagger$ & $8+$ \\
\hline Intervention arms & 55 & 46 & 9 \\
\hline \multicolumn{4}{|l|}{ Participants enrolled: } \\
\hline Total & 8406 & 7687 & 1031 \\
\hline Median within individual trials (min-max) & $133(16-1134)$ & $133(16-1134)$ & $118(50-235)$ \\
\hline \multicolumn{4}{|l|}{ Meta-analyses to which each trial contributed: } \\
\hline Median maternal outcomes (min-max) & $2(0-6)$ & $2(0-6)$ & $2(0-4)$ \\
\hline Median newborn/infant outcomes (min-max) & $3(0-17)$ & $3(0-17)$ & $4(0-7)$ \\
\hline Trials that did not contribute data to any meta-analyses & $2(4.7)$ & $2(5.4)$ & $0(0.0)$ \\
\hline \multicolumn{4}{|l|}{ Geographical region: } \\
\hline Europe & $7(16.3)$ & $7(18.9)$ & $2(25.0)$ \\
\hline South East Asia & $6(14.0)$ & $2(5.4)$ & $4(50.0)$ \\
\hline Americas & $5(11.6)$ & $5(13.5)$ & $0(0.0)$ \\
\hline Eastern Mediterranean & $21(48.8)$ & $19(51.4)$ & $2(25.0)$ \\
\hline Western Pacific & $4(9.3)$ & $4(10.8)$ & $0(0.0)$ \\
\hline \multicolumn{4}{|l|}{ Health status at enrolment: } \\
\hline Generally healthy & $37(86.0)$ & $33(89.2)$ & $6(75.0)$ \\
\hline Gestational diabetes & $4(9.3)$ & $2(5.4)$ & $2(25.0)$ \\
\hline Hypocalcaemia & $1(2.3)$ & $1(2.7)$ & \\
\hline Multiple sclerosis & $1(2.3)$ & $1(2.7)$ & $0(0.0)$ \\
\hline \multicolumn{4}{|l|}{ Baseline vitamin D status $\neq:$} \\
\hline Mean $25(\mathrm{OH}) \mathrm{D}<30 \mathrm{nmol} / \mathrm{L}$ & $10(23.3)$ & $9(24.3)$ & $2(25.0)$ \\
\hline Mean $25(\mathrm{OH}) \mathrm{D} \geq 30 \mathrm{nmol} / \mathrm{L}$ & $23(53.5)$ & $20(54.1)$ & $3(37.5)$ \\
\hline Not reported & $10(23.3)$ & $8(21.6)$ & $3(37.5)$ \\
\hline \multicolumn{4}{|l|}{ Type of vitamin D\&: } \\
\hline $\mathrm{D} 2$ & $4(7.3)$ & $3(6.5)$ & $1(11.1)$ \\
\hline D3 & $48(87.3)$ & $40(87.0)$ & $8(88.9)$ \\
\hline Not reported & $3(5.5)$ & $3(6.5)$ & $0(0.0)$ \\
\hline \multicolumn{4}{|l|}{ Supplementation frequency§: } \\
\hline Daily & $31(56.4)$ & $31(67.4)$ & - \\
\hline Weekly & $5(9.1)$ & $5(10.9)$ & - \\
\hline Every 2 weeks & $6(10.9)$ & $6(13.0)$ & - \\
\hline Monthly & $3(5.5)$ & $3(6.5)$ & - \\
\hline Every 2 months & $1(1.8)$ & $1(2.2)$ & - \\
\hline Single dose & $3(5.5)$ & - & $3(33.3)$ \\
\hline Two doses & $4(7.3)$ & - & $4(44.4)$ \\
\hline Other & $2(3.6)$ & - & $2(22.2)$ \\
\hline \multicolumn{4}{|l|}{ Intervention dose§ף: } \\
\hline Median (min-max) & $2000(200-7543)$ & $2000(200-7543)$ & - \\
\hline Regular dose $<2000 \mathrm{IU} /$ day & $24(52.2)$ & $24(52.2)$ & - \\
\hline Regular dose $\geq 2000 \mathrm{IU} /$ day & $22(47.8)$ & $22(47.8)$ & - \\
\hline \multicolumn{4}{|l|}{ Control intervention type: } \\
\hline Placebo & $29(67.4)$ & $23(62.2)$ & $8(100.0)$ \\
\hline Active & $14(32.6)$ & $14(37.8)$ & $0(0.0)$ \\
\hline Median vitamin D dose in active control arms (min-max) & $400(200-600)$ & $400(200-600)$ & - \\
\hline \multicolumn{4}{|l|}{ Timing of initiation of supplementation§: } \\
\hline 1st trimester & $19(34.5)$ & $18(39.1)$ & $1(11.1)$ \\
\hline 2nd trimester & $30(54.5)$ & $24(52.2)$ & $6(66.7)$ \\
\hline 3rd trimester & $5(9.1)$ & $3(6.5)$ & $2(22.2)$ \\
\hline Not reported & $1(1.8)$ & $1(2.2)$ & $0(0.0)$ \\
\hline \multicolumn{4}{|c|}{$\begin{array}{l}\text { *Regular dosing refers to regimens in which supplement was administered at least three times at regular frequency throughout pregnancy (daily, } \\
\text { weekly, every } 2 \text { weeks, monthly, or every } 2 \text { months); bolus dosing refers to interventions administered either once or twice during intervention } \\
\text { period. } \\
\text { †Two trials (Mallet } 1986 \text { and Yu 2009, see table A in appendix 5) are represented in both columns because they included both regular and bolus } \\
\text { dose intervention arms. } \\
\text { fMean baseline maternal serum 25(OH)D concentration in control group (that is, at enrolment). } \\
\text { §Characteristic defined for each intervention arm; therefore, denominator is "intervention arms" rather than "trials." } \\
\text { १Regular doses given at frequencies other than daily expressed as equivalent daily doses. "Monthly" doses divided by } 30.4 \text { to estimate equivalent } \\
\text { daily doses. }\end{array}$} \\
\hline
\end{tabular}

on published trials methods) or reported in qualitative terms or a format that could not be incorporated into a meta-analysis (appendix 6). Five trials met the broadest two criteria for inclusion in the systematic review but were ineligible for inclusion in metaanalyses (appendix 7).

\section{Characteristics and quality of trials included in meta-analyses}

The 43 eligible trials comprised 55 interventioncontrol arm comparisons and a total of 8406 enrolled women (table 1; tables A and B in appendix 5). Most trials were conducted in 2000 or later (88\%), with an 
acceleration in the publication rate after about 2010, particularly in the Eastern Mediterranean region (fig 2). Few trials in South Asia were published recently, and there were no trials in Africa (table 1; fig 2). For most outcomes, meta-analyses included data from a minority of the trials, and two trials did not contribute to any meta-analyses (appendix 8). Several trials mentioned outcomes in a manner that did not meet minimum criteria for case defintition and/or methods of ascertainment and were thus included only in sensitivity analyses (table $C$ in appendix 5). There was substantial clinical and methodological heterogeneity between trials (table 1), including wide variation in baseline maternal vitamin D status (fig 3; table B in appendix 5). Among regular dose trials, effective doses were no higher in populations with lower baseline vitamin D status, and placebo controlled trials did not consistently use higher effective doses nor were they more likely to be conducted in populations deficient in vitamin D compared with active control trials (fig 3). Only eight of 43 trials (19\%) had an overall low risk of bias (appendix 9).

\section{Effects on maternal and cord blood}

\section{5-hydroxyvitamin D concentrations}

We performed meta-analyses for 11 maternal and 27 neonatal/infant outcomes (tables 2 and 3). The most widely reported outcome was mean maternal $25(\mathrm{OH})$ $\mathrm{D}$ concentration at or near delivery, and data on cord 25(OH)D concentration were also often available

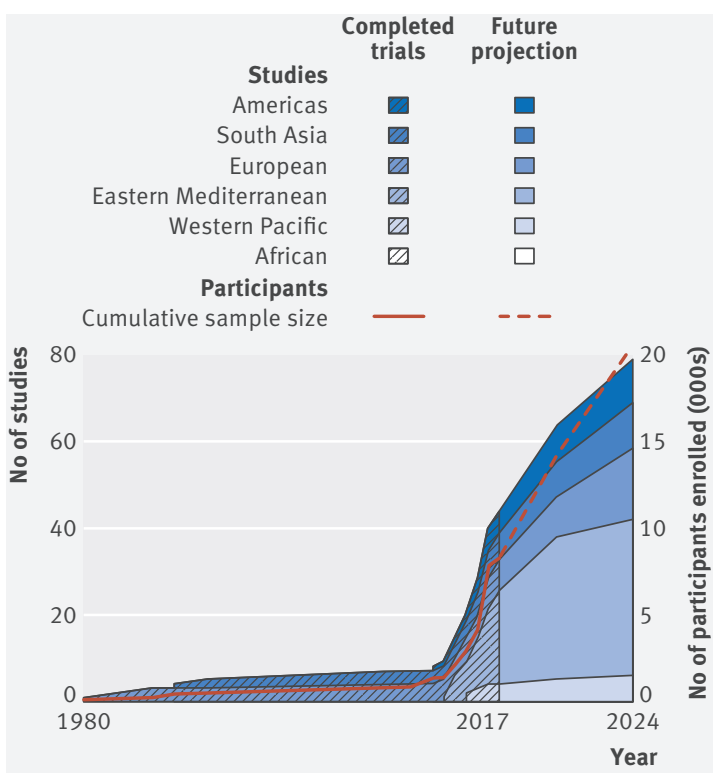

Fig 2 | Accumulation of prenatal vitamin D supplementation trials 1980-2024. Bands represent number of trials in each geographical region. Hatched areas and solid line are based on completed and published trials up until September 2017. Non-hatched areas and broken line represent projections from September 2017 to 2024 for future/ongoing trials identified in clinical trial registries. No published trials were conducted in Africa, though one large trial in Tanzania $(n=2300)$ is ongoing and projected to be completed and published before 2024 (table 3; appendix 7). Overall, intervention groups attained significantly higher maternal and cord 25(OH) D concentrations than the control groups (table 3 ). Higher regular dose regimens ( $\geq 2000 \mathrm{IU} /$ day) led to a greater average increment in maternal delivery and cord 25(OH)D than lower doses (tables S1 and S2 in appendix 10); however, the linear dose-response curves for maternal and cord blood indicated modest effects of increasing vitamin $\mathrm{D}$ doses on the increment in 25(OH)D concentrations (fig S2 and S4 in appendix 10). The 25(OH)D response to supplemental vitamin D was not enhanced in trial populations with low mean baseline 25(OH)D (<30 nmol/L) (tables S1 and S2 in appendix 10).

\section{Effects on maternal clinical outcomes and adverse events}

Maternal clinical outcomes were reported in only a few small trials (table 2; tables S3-S12 and figs S5-S26 in appendix 10). Pooled effect estimates therefore had wide confidence intervals except for caesarean section (for which there was evidence of no effect of vitamin D), and 95\% confidence intervals for all primary pooled estimates for maternal clinical outcomes except hypocalcaemia included the null (table 2). Numerous trials mentioned pre-eclampsia, gestational hypertension, or gestational diabetes in a manner that did not meet our minimum criteria for case definitions and methods of ascertainment (tables S3-S5 in appendix 10). Inclusion of all trials irrespective of these criteria, however, suggested that vitamin D could reduce the risk of gestational diabetes (risk ratio $0.61,95 \%$ confidence interval 0.45 to 0.83 ; 14 comparisons; 2643 participants)

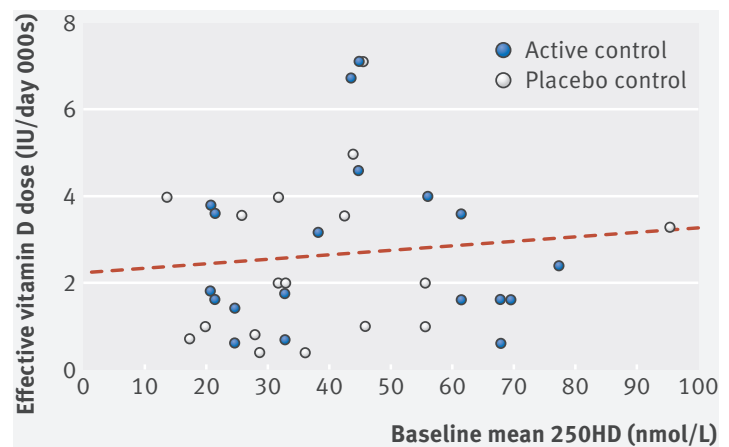

Fig 3 | Effective equivalent daily vitamin D dose (IU/day) versus maternal baseline mean 25 -hydroxyvitamin $D$ $(25(\mathrm{OH}) \mathrm{D})$ concentration $(\mathrm{nmol} / \mathrm{L})$ in published prenatal vitamin D supplementation trials with regular dosing regimens (29 trials; 36 comparisons). "Effective" dose is dose administered in intervention group minus dose in control group (200-600 IU/day in active-control trials; 0 $\mathrm{IU} /$ day in placebo control trials). Each point represents intervention-control comparison from single trial. Linear trend line fitted with generalised estimating equations to account for correlations within trials for trials with more than one intervention arm. Estimated mean change in effective vitamin $D$ dose for every $20 \mathrm{nmol} / \mathrm{L}$ increase in baseline mean 25(OH)D concentration was increase of $208 \mathrm{IU} /$ day $(\mathrm{P}=0.54)$ 


\begin{tabular}{|c|c|c|c|c|c|c|}
\hline Outcomes & $\begin{array}{l}\text { Trials reporting } \\
\text { eligible outcome }\end{array}$ & $\begin{array}{l}\text { Trials } \\
\text { included* }\end{array}$ & $\begin{array}{l}\text { Intervention-control } \\
\text { comparisons included* }\end{array}$ & $\begin{array}{l}\text { Participants } \\
\text { included* }\end{array}$ & Risk ratio $(95 \% \mathrm{Cl})$ & $I^{2}(\%)$ \\
\hline \multicolumn{7}{|l|}{ Maternal } \\
\hline Pre-eclampsia & 3 & 3 & 3 & 706 & $1.09(0.43$ to 2.76$)$ & $67 \dagger$ \\
\hline Gestational hypertension & 2 & 2 & 2 & 564 & 1.69 (0.73 to 3.92) & 0 \\
\hline Gestational diabetes & 5 & 5 & 5 & 1030 & 0.65 (0.39 to 1.08$)$ & 45 \\
\hline Stillbirth & 16 & 16 & 19 & 4606 & $0.75(0.51$ to 1.13$)$ & 0 \\
\hline Caesarean section & 17 & 16 & 18 & 3240 & $1.02(0.93$ to 1.12$)$ & 0 \\
\hline Preterm labor & 4 & 4 & 4 & 776 & $0.52(0.26$ to 1.05$)$ & 51 \\
\hline Hypercalcaemia & 4 & 1 & 1 & 175 & $3.11(0.87$ to 11.08$)$ & - \\
\hline Hypercalciuria & 2 & 1 & 1 & 160 & $3.00(0.12$ to 72.56$)$ & - \\
\hline Hypocalcaemia & 2 & 1 & 1 & 200 & $0.05+(0.01$ to 0.18$)$ & - \\
\hline Admission to hospital & 5 & 5 & 5 & 1776 & $0.97(0.69$ to 1.35$)$ & 0 \\
\hline \multicolumn{7}{|l|}{ Neonatal/infant } \\
\hline Preterm birth & 14 & 13 & 15 & 3757 & $1.00(0.77$ to 1.30$)$ & 0 \\
\hline Low birth weight & 8 & 7 & 7 & 1156 & $0.74(0.47$ to 1.16$)$ & 47 \\
\hline Small for gestational age & 5 & 5 & 7 & 741 & $0.60+(0.40$ to 0.90$)$ & 0 \\
\hline Congenital malformations & 5 & 4 & 4 & 2509 & $0.93(0.61$ to 1.42$)$ & 0 \\
\hline Neonatal death & 5 & 3 & 4 & 1202 & $0.48(0.16$ to 1.49$)$ & 0.0 \\
\hline Hypercalcaemia & 1 & 1 & 1 & 73 & 0.97 (0.31 to 3.08) & - \\
\hline Hypocalcaemia & 2 & 1 & 1 & 126 & $0.10(0.01$ to 1.83$)$ & - \\
\hline Admission to neonatal intensive care unit & 5 & 5 & 6 & 1997 & $0.92(0.66$ to 1.28$)$ & 21 \\
\hline Respiratory infections (general) & 1 & 1 & 2 & 223 & $0.97(0.77$ to 1.23$)$ & 0 \\
\hline Upper respiratory tract infections & 2 & 2 & 4 & 389 & $0.91(0.77$ to 1.06$)$ & 0 \\
\hline Lower respiratory tract infections & 4 & 4 & 6 & 1769 & $0.97(0.84$ to 1.12$)$ & 0 \\
\hline Asthma or recurrent/persistent wheeze by age 3 years & 2 & 2 & 2 & 1387 & $0.81+(0.67$ to 0.98$)$ & 0 \\
\hline
\end{tabular}

(table S5 and fig S10 in appendix 10). In contrast, ignoring these criteria did not change inferences with respect to pre-eclampsia (0.82, 0.63 to 1.07 ; 16 comparisons; 3398 participants) or gestational hypertension $(0.83,0.53$ to $1.30 ; 8$ comparisons; 2430 participants) (tables S3-S4 and fig S6 and S8 in appendix 10). Biochemical safety outcomes (hypercalcaemia, hypercalciuria, hypocalcaemia) were rare; only four trials met our minimum criteria for case definitions and methods of ascertainment based on routine monitoring of maternal serum calcium (table 2; tables S9-S11in appendix 10), yet ignoring the these criteria did not change the inference that there was no significant effect of vitamin D supplementation on the risk of hypercalcaemia, which is a primary indicator of toxicity of vitamin D supplementation (table S9 and fig S18 in appendix 10).

\begin{tabular}{|c|c|c|c|c|c|}
\hline Outcomes & Trials & $\begin{array}{l}\text { Intervention-control } \\
\text { comparisons }\end{array}$ & Participants & $\begin{array}{l}\text { WMD (intervention versus } \\
\text { control) }(95 \% \mathrm{Cl})\end{array}$ & $\begin{array}{l}\mathrm{I}^{2} \\
(\%)\end{array}$ \\
\hline \multicolumn{6}{|l|}{ Maternal } \\
\hline Serum $25(\mathrm{OH}) \mathrm{D}$ concentration at/near delivery $(\mathrm{nmol} / \mathrm{L})$ & 32 & 42 & 5706 & $32.91^{*}(27.19$ to 38.62$)$ & $96^{*}$ \\
\hline \multicolumn{6}{|l|}{ Neonatal/infant } \\
\hline Birth weight (g) & 30 & 37 & 5273 & $58.33^{*}(18.88$ to 97.78$)$ & $43^{*}$ \\
\hline Birth length $(\mathrm{cm})$ & 19 & 21 & 3899 & $0.19(-0.08$ to 0.47$)$ & $62^{\star}$ \\
\hline Head circumference at birth $(\mathrm{cm})$ & 17 & 18 & 3791 & $0.13(-0.05$ to 0.30$)$ & $63^{*}$ \\
\hline Cord serum 25(OH)D concentration $(\mathrm{nmol} / \mathrm{L})$ & 20 & 28 & 2988 & $27.73^{*}(21.57$ to 33.88$)$ & $96^{\star}$ \\
\hline Gestational age at birth (weeks) & 19 & 23 & 3214 & $-0.01(-0.19$ to 0.16$)$ & $54^{*}$ \\
\hline Neonatal bone mineral content (g) & 2 & 2 & 690 & $1.09(-0.64$ to 2.81$)$ & 0 \\
\hline Infant bone mineral content (g) & 1 & 2 & 52 & $-43.00^{*}(-67.25$ to -18.75$)$ & 0 \\
\hline Neonatal bone mineral density $\left(\mathrm{g} / \mathrm{cm}^{2}\right)$ & 2 & 2 & 690 & $0.00(-0.003$ to 0.004$)$ & 0 \\
\hline Infant bone mineral density $\left(\mathrm{g} / \mathrm{cm}^{2}\right)$ & 1 & 2 & 52 & $-0.04^{*}(-0.06$ to -0.03$)$ & 0 \\
\hline Length at age 1 year $(\mathrm{cm})$ & 2 & 2 & 251 & $1.30 *(0.54$ to 2.06$)$ & 40 \\
\hline Weight at age 1 year (g) & 2 & 2 & 252 & $290.62(-5.04$ to 586.29$)$ & 47 \\
\hline Head circumference at age 1 year $(\mathrm{cm})$ & 2 & 2 & 248 & $0.09(-0.28$ to 0.45$)$ & 0 \\
\hline Length for age $z$ score at 1 yeart & 2 & 3 & 186 & $0.31(-0.04$ to 0.66$)$ & 0 \\
\hline Weight for age $z$ score at 1 yeart & 2 & 3 & 187 & $0.13(-0.19$ to 0.45$)$ & 0 \\
\hline Head circumference for age $z$ score at 1 yeart & 2 & 3 & 183 & $0.12(-0.18$ to 0.42$)$ & 0 \\
\hline
\end{tabular}


Effects on fetal growth and preterm birth

Birth anthropometry and preterm birth were among the most commonly reported outcomes (tables 2 and 3). Pooling of 37 comparisons indicated that prenatal vitamin D supplementation (versus low dose, no vitamin $\mathrm{D}$, or placebo) increased mean birth weight by an average of $58 \mathrm{~g}$ (fig 4). In a cumulative metaanalysis, 95\% confidence intervals have excluded the null since 2013, but the magnitude of the effect on birth weight attenuated over the most recent few years (fig 4). The magnitude of the pooled effect remained relatively stable in sensitivity analyses (table S13 in appendix 10) and was unaffected by the removal of single outlier trials (not shown). There was significant heterogeneity between trials that might have been partly explained by the greater effects on birth weight in groups that received bolus doses of vitamin D3 and in trials that were conducted in South Asia (table S13 in appendix 10). Furthermore, there was no significant dose-response effect or inverse relation with baseline $25(\mathrm{OH}) \mathrm{D}$ (fig S28 in appendix 10). In a post hoc analysis of the effect on birth weight that took into account both effective dose and baseline vitamin D status, there was a significant dose-response effect in trials in which mean baseline 25(OH)D was $30-50 \mathrm{nmol} / \mathrm{L}$ but no association in trials with mean 25(OH)D <30 nmol/L (fig S29 in appendix 10).
A

\section{Study}

Mallet (H) (1986)

Khan (2016)

Shahgheibi (2016)

Thiele (2016)

Mallet (L) (1986)

Mutlu (H) (2014)

Vaziri (2016)

Charandabi (2015)

Cooper (2016)

Mojibian (2015)

Litonjua (2016)

Sahoo (H) (2016)

Dawodu (H) (2013)

Roth (2013)

Yu (H) (2009)

Mutlu (L) (2014)

Grant (L) (2014)

Abotorabi (2017)

Sabet (2012)

Yu (L) (2009)

Hossain (2014)

Hollis (H) (2011)

Yap (2014)

Grant (H) (2014)

Dawodu (L) (2013)

Sahoo (L) (2016)

Asemi (b) (2013)

Brooke (1980)

Hollis (L) (2011)

Hashemipour (2013)

Karamali (2015)

Marya (1988)

Sablok (2015)

Valizadeh (2016)

Naghshineh (2016)

Zerofsky (2014)

Kaur (1991)

Overall

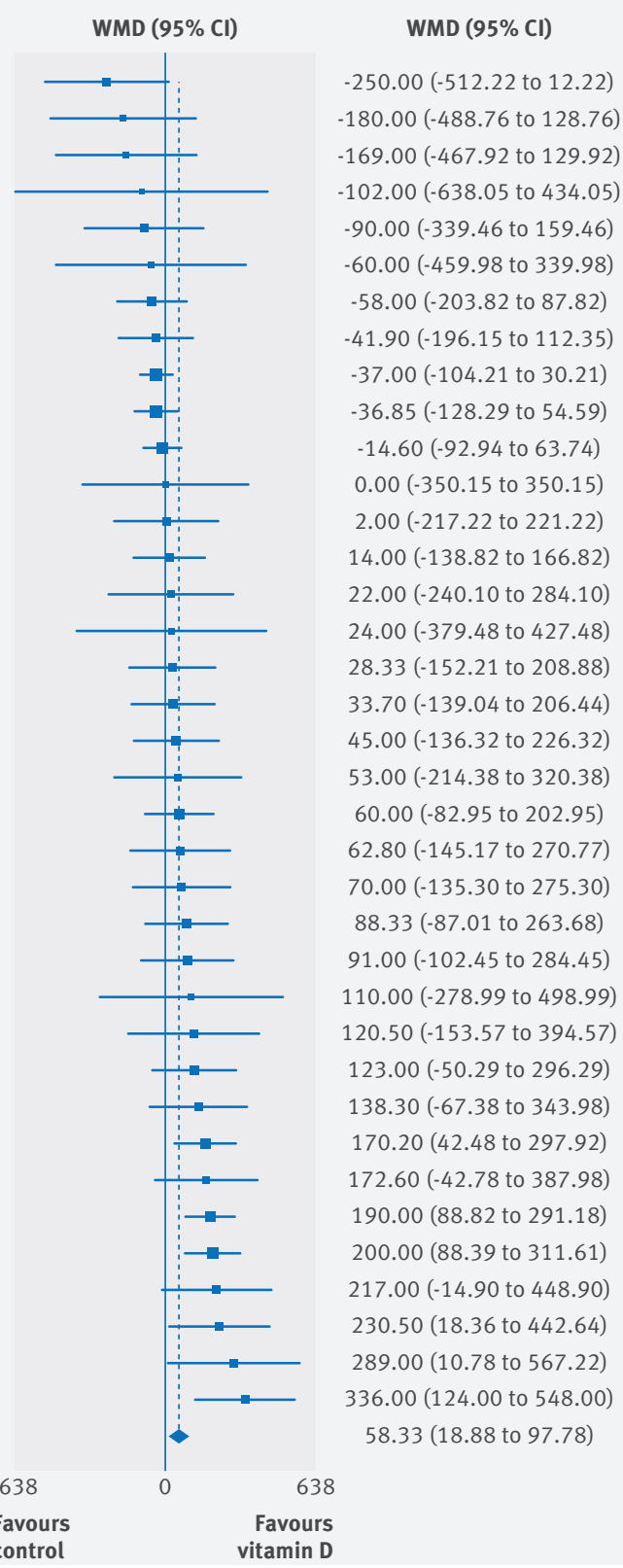

B

Study

Brooke (1980)

Mallet (H) (1986)

Mallet (L) (1986)

Marya (1988)

Kaur (1991)

Yu (H) (2009)

Yu (L) (2009)

Hollis (H) (2011)

Hollis (L) (2011)

Sabet (2012)

Asemi (b) (2013)

Dawodu (H) (2013)

Dawodu (L) (2013)

Hashemipour (2013)

Roth (2013)

Grant (H) (2014)

Grant (L) (2014)

Hossain (2014)

Mutlu (H) (2014)

Mutlu (L) (2014)

Yap (2014)

Zerofsky (2014)

Charandabi (2015)

Karamali (2015)

Mojibian (2015)

Sablok (2015)

Cooper (2016)

Khan (2016)

Litonjua (2016)

Naghshineh (2016)

Sahoo (H) (2016)

Sahoo (L) (2016)

Shahgheibi (2016)

Thiele (2016)

Valizadeh (2016)

Vaziri (2016)

Abotorabi (2017)

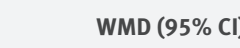

WMD $(95 \% \mathrm{Cl})$

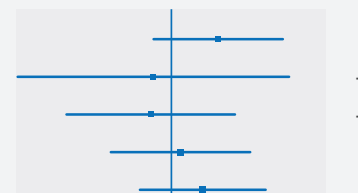

123.00 ( -50.29 to 296.29$)$

49.98 (-414.56 to 314.59$)$

54.95 (-278.79 to 168.88$)$

22.28 (-162.79 to 207.36$)$

82.62 (-84.34 to 249.59$)$

76.29 (-69.59 to 222.17$)$

75.85 ( -53.26 to 204.97$)$

$76.94(-35.72$ to 189.60$)$

86.61 (-12.72 to 185.95$)$

83.90 ( -5.10 to 172.90$)$

88.02 (5.55 to 170.48 )

82.08 (4.62 to 159.54 )

84.81 (14.06 to 155.56 )

96.40 (32.26 to 160.55 )

89.33 (28.62 to 150.03 )

90.84 (34.54 to 147.15 )

87.72 (34.34 to 141.10 )

86.80 (37.27 to 136.33 )

85.28 (36.72 to 133.83 )

85.65 (38.61 to 132.68 )

86.51 (41.88 to 131.14 )

90.33 (45.26 to 135.39 )

80.68 (35.44 to 125.93 )

84.37 (40.50 to 128.23 )

71.49 (26.50 to 116.48 )

80.19 (35.16 to 125.22$)$

70.48 (24.66 to 116.31 )

66.08 (20.19 to 111.97 )

60.65 (17.06 to 104.25 )

65.43 (21.94 to 108.93 )

64.53 (21.74 to 107.31 )

64.92 (22.77 to 107.08 )

61.25 (19.13 to 103.37)

60.31 (18.59 to 102.02 )

63.88 (22.43 to 105.33 )

59.20 (18.56 to 99.84 )

58.33 (18.88 to 97.78 )

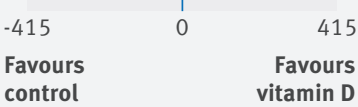

Fig 4 | A: Weighted mean differences (WMDs) and B: cumulative WMDs for effect of prenatal vitamin D supplementation on mean birth weight (g), estimated with random effects meta-analysis, based on 37 intervention-control comparisons in 30 randomised controlled trials of regular or bolus regimen vitamin D at any dose in studies published by September 2017 
Consistent with the pooled effect on mean birth weight-but in a smaller subset of only seven comparisons-vitamin D reduced the risk of small for gestational age (SGA) (table 2; table S14 and fig S30 in appendix 10). Although there was little heterogeneity across trials and a lack of notable subgroup effects, none of the studies that reported data on small for

Key

Reported and eligible (met minimum criteria for case definition and methods of ascertainment)

$\square$ Reported but did not meet minimum criteria for case definition and methods of ascertainment

$\square$ Reported but in manner that prevented inclusion in meta-analysis

$\square$ Unreported because of loss of follow-up or incomplete reporting

$\square$ Missing (not ascertained and/or not reported)

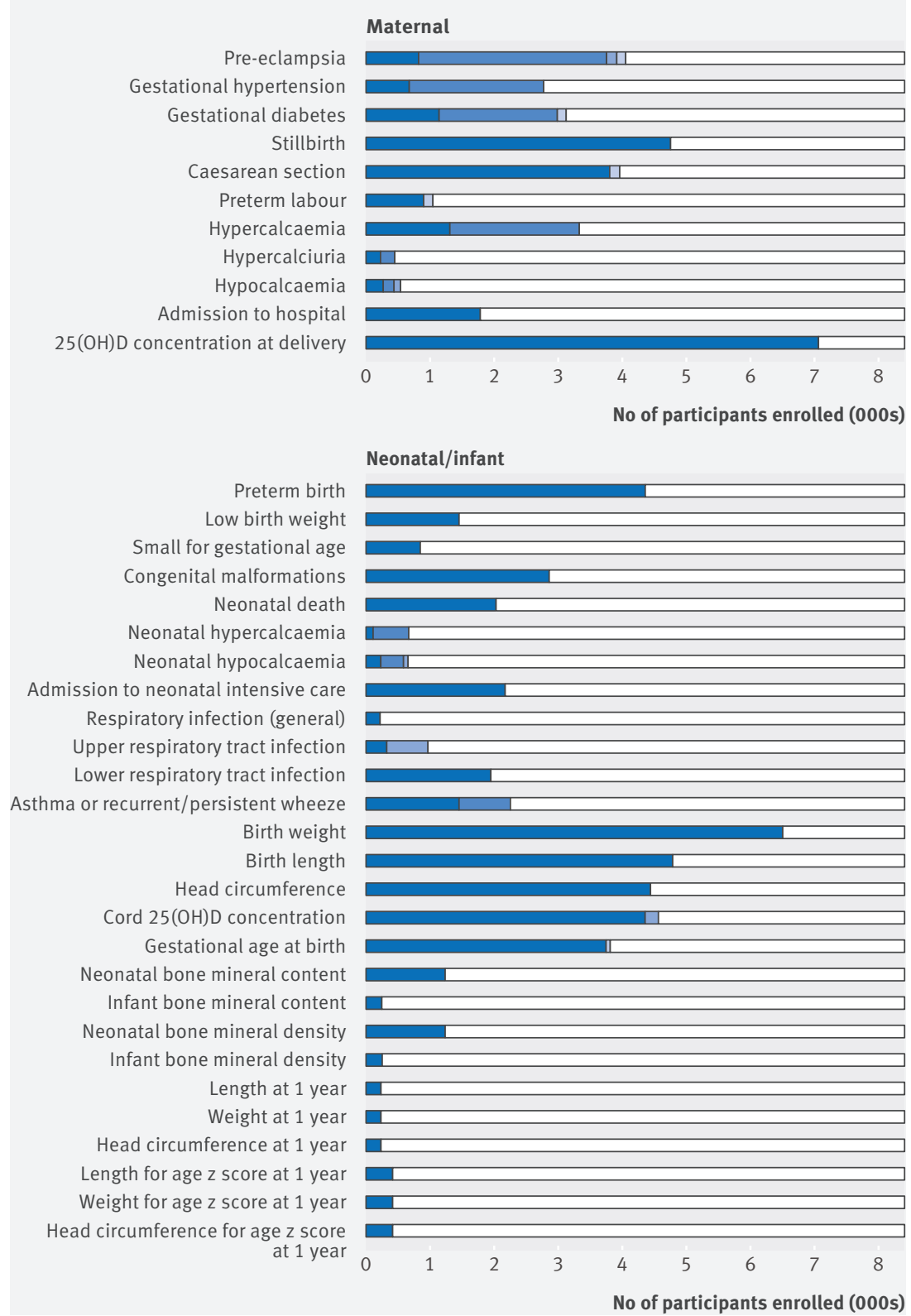

Fig 5 | Proportion of all participants $(n=8406)$ enrolled in eligible trials for which maternal and neonatal/infant outcomes were reported and met criteria for case definition and method of ascertainment; reported but did not meet minimum criteria; reported, but in manner that prevented inclusion of data in meta-analysis; missing because of loss of follow-up in trials for which outcome was reported for some participants or because of apparent incomplete reporting of planned outcomes; or missing because outcome was not assessed or not mentioned in published reports gestational age had an overall low risk of bias (table S14 in appendix 10). The available evidence did not indicate a significant effect on other indicators of fetal growth, including low birth weight, birth length, or birth head circumference (table 3; tables S15-S17 and figs S31-S36 in appendix 10). There was no apparent effect of vitamin D on gestational age at birth (table 3; table S18 and figs S37-S38 in appendix 10) or the risk of preterm birth (table 2; table S19 and figs S39-S41 in appendix 10).

\section{Effects on newborn and infant clinical outcomes and adverse events}

Clinical events in newborns (congenital anomalies, admission to neonatal care unit, death) as well as neonatal hypocalcaemia or hypercalcaemia were infrequently and inconsistently reported (table 2; tables S20-S24 and figs S42-S50 in appendix 10). Infant clinical or functional outcomes beyond the newborn period were also rarely reported. The pooled estimate from two high quality regular dose trials in high income countries (total 1387 participants) indicated that prenatal vitamin D supplementation significantly decreased the risk of persistent/recurrent wheeze in offspring by age 3 years (table 2; fig S51 in appendix 10). This inference remained unchanged on inclusion of three other trials for which asthma/wheeze outcomes did not meet our minimum criteria for case definitions and methods of ascertainment (table S25 and fig S52 in appendix 10). There were no significant effects on the risk of infant acute respiratory infections overall, risk of ever having an upper respiratory infection, or the risk of ever having a lower respiratory infections (table 2; tables S26-S28 and figs S53-S55 in appendix 10).

Two studies reported effects on neonatal bonemineral content and bone mineral density, for which the metaanalysis did not support an effect of vitamin D (table 3; tables S29-S30 and figs S56-S57 in appendix 10). One trial (two comparisons) found that bone mineral content and density at 12-16 months of age were lower in the vitamin D groups than in placebo groups (table 3; tables S31-S32 and figs S58-S59 in appendix 10). Two trials in which infant anthropometry was reported with raw measures showed an increase in length at age 1 year, but the overall effect on mean length for age $\mathrm{z}$ score in two studies (three comparisons) was not significant (table 3; tables S33 and S36 and figs S60 and S63 in appendix 10). There were no significant pooled effects on other anthropometric measures in infancy (table 3; tables S34-S35 and S37-S38 and figs S61-S62 and S64-S65 in appendix 10).

\section{Missing outcomes}

Maternal and cord 25(OH)D at delivery, stillbirth, preterm birth, and birth weight/length/head circumference were the only outcomes reported for more than half of enrolled participants (fig 5; appendix 6). As most women were enrolled in trials that apparently did not implement surveillance for other clinical outcomes, outcome data were often unavailable (fig 5). For some maternal outcomes (such as pre-eclampsia, hypertension, gestational diabetes, maternal hypercalcaemia), a 
substantial number of participants were enrolled in trials in which such outcomes were mentioned but could not be included in the primary meta-analyses because they did not meet a minimum set of criteria for case definitions and methods of ascertainment (fig 5; table C in appendix 5). A relatively small minority of participants were excluded because of loss to follow-up or because outcomes were reported but in a manner that could not be technically included in the meta-analysis (fig 5).

Future projections of evidence based on registered ongoing/planned trials

We identified 35 registered trials of prenatal vitamin D with a total target enrolment of 12530 participants, of which 19 (54\%) were completed but unpublished, $11(31 \%)$ were ongoing or planned, and five (14\%) had indeterminate status (fig 2; table A and fig A in appendix 11). Of the 35 registered trials, $22(63 \%)$ have either a non-intervention or placebo control group and most (74\%) involve regular dosing regimens ranging from $400 \mathrm{IU} /$ day to $4000 \mathrm{IU} /$ day. Among trials with bolus regimens, doses varied from $400 \mathrm{IU}$ to 300000 IU. Nearly half of the trials (40\%) are in the Eastern Mediterranean, of which 10 are in Iran. Most (57\%) are in low or middle income countries, including Bangladesh, India, Pakistan, Mongolia, and Tanzania. The largest trial involves 2300 HIV positive women in Tanzania (NCT02305927), with the primary outcomes of maternal progression of HIV, small for gestational age, and stunting in infancy. The second largest trial, involving 1300 women in Bangladesh (NCT01924013), was designed to assess effects on infant mean length for age $\mathrm{z}$ score at one year, with a substudy focused on the effects on infant viral acute respiratoy infections in the first six months of life (NCT02388516). The third largest trial, involving 1000 women in Sweden (2010-019483-37), has numerous outcomes including pre-eclampsia, preterm labour, preterm birth, low birth weight, and stillbirth. Similar to published trials, most of the ongoing/planned trials do not seem to have been designed to generate inferences related to clinical outcomes. The most common trial outcomes will be maternal 25(OH)D at delivery, cord 25(OH)D, and hypertensive diseases (table B in appendix 11). Optimistic projections indicate that 15 trials (6610 participants) will contribute data related to hypertensive disorders (preeclampsia and gestational hypertension) (table B in appendix 11), but detailed criteria for case definitions and methods of ascertainment were rarely described in registry entries. Only two trials (1740 participants) will report infant acute respiratoy infection outcomes. No registered trials indicated neonatal/infant mortality or rickets as outcomes.

\section{Discussion}

Though trials of prenatal vitamin D supplementation are being published at an accelerating pace, randomised controlled trials published up to 2017 were generally small, low quality, and rarely designed to examine clinical outcomes. Although data could be statistically pooled for several outcomes, irreconcilable heterogeneity between trials (such as variable baseline vitamin D status) rendered most inferences difficult to interpret or apply. Moreover, missing data on clinical outcomes was the norm rather than exception, leading to potentially biased meta-analyses based on small non-representative subsets of trials and participants.

Though some observational studies have shown associations between maternal vitamin D deficiency and gestational diabetes and pre-eclampsia, ${ }^{22}$ we did not find robust corroborating evidence from randomised controlled trials. Few trials, however, deliberately measured maternal conditions related to pregnancy or adverse events. Most trials reported mean birth weight, providing a wide distribution of estimates surrounding the pooled effect of $+58 \mathrm{~g}$ in the vitamin D groups. This could be evidence of the biological responsiveness of fetal growth to vitamin D, consistent with observational epidemiological studies $^{22} 23$ and some animal research. ${ }^{24} 25$ Yet this is also an example of an intangible meta-analysis result that frustrates as much as it illuminates as the effect cannot be attributed to a specific dose or target 25(OH)D threshold. No large single trial produced the effect size and inference implied by the meta-analysis, and subgroup analyses and meta-regression mostly weakened the inference. Moreover, there were no significant effects on other neonatal anthropometric variables. Two trials indicated that vitamin D could enhance postnatal linear growth, an outcome related to growth plates for which a hypothesised role of vitamin $\mathrm{D}$ is logical, ${ }^{26}$ and which is the primary focus of our as yet unpublished trial in Bangladesh. ${ }^{27}$ In the few studies reporting effects on infant bone mass, results were mixed; the largest of these trials-the MAVIDOS study in the UK, which excluded women with $25(\mathrm{OH})$ D concentrations $<25 \mathrm{nmol} / \mathrm{L}^{28}$-has scheduled followup of children at age 4 years. ${ }^{29}$ Infant rickets was not reported in any trials; though rare, this is one of the most likely paediatric conditions to be directly caused by poor maternal and early infant vitamin D status. ${ }^{30}$ Adverse events were infrequently reported, resulting in unreliable meta-analyses. For example, prenatal vitamin D supplementation in deficient women modifies neonatal calcium homeostasis, ${ }^{31}$ but clinical correlates (such as symptomatic infant hypocalcaemia) were rare and infrequently ascertained across trials. Nonetheless, there was no evidence of any specific harms to mothers or fetuses attributable to vitamin D supplementation within the range of reported doses.

One of the most robust findings in our systematic review-which has been previously reported ${ }^{32}$-was the beneficial effect of prenatal vitamin D on risk of persistent/recurrent wheeze (or asthma) in offspring, based on data from two large well conducted trials in which wheeze/asthma was the primary outcome. ${ }^{33} 34$ This is broadly consistent with the possibly positive effect of vitamin D on severity of illness in adults with asthma. ${ }^{5}$ Before prenatal vitamin $\mathrm{D}$ can be recommended as a routine preventive measure against childhood asthma, however, further work is required 
to clarify the minimum effective dose and longer term follow-up to determine if observed early effects on symptoms translate into reductions in the incidence of asthma and improvements in pulmonary function.

Meta-regression or subgroup analyses can theoretically illustrate dose-responsiveness, thereby more precisely guide supplement dosing. The interpretation of micronutrient intervention trials, however, is complex because all individuals have some vitamin $\mathrm{D}$ intake or endogenous production. ${ }^{35}$ The effect of a given dose depends on the difference between the intervention and control doses (or the difference in mean 25(OH)D in the intervention versus control group at/near delivery) as well as baseline vitamin D status. Populations without vitamin D deficiency might have little to gain from any dose, but deficient populations might require relatively high doses to raise vitamin D status to an optimal range associated with clinical benefits. ${ }^{35}$ In this framework, the distinction between placebo versus active control trials is only informative insofar as an active control dose increases the baseline status to which the intervention dose is compared (as such, we considered a "modified" baseline status to be reflected by the mean 25(OH)D at delivery in the control group). If a modest dose of vitamin D ( $<600 \mathrm{IU} /$ day) readily eliminates deficiency in even the most deficient women, then we might expect to see benefits only in placebo control trials conducted in populations where deficiency is common. Overall, the present findings did not adhere to these expected or plausible doseresponse patterns; specifically, we did not consistently find greater effects at higher effective doses, when there were larger differences in maternal 25(OH)D at delivery, in relatively deficient populations (based on either initial or modified baseline), or in placebo controlled trials. Many of the reported doses, however, did not yield effects on maternal 25(OH)D that would be expected based on vitamin $\mathrm{D}$ pharmacokinetics. ${ }^{3637}$ The muted dose-25(OH)D relation could be attributed to high baseline $25(\mathrm{OH}) \mathrm{D}$, low precision of $25(\mathrm{OH})$ D assays, low rates of adherence to supplementation regimens, or lower than labeled vitamin $\mathrm{D}$ content of the supplements. We considered the weak association between dose and 25(OH)D to reflect the overall low quality of many of the trials, which tempered expectations of finding dose dependent effects of vitamin D on clinical outcomes.

Compared with the 2016 Cochrane review by DeRegil and colleagues, ${ }^{6}$ we included all nine trials that were included in the Cochrane review, 10 trials that were published before June 2015 (when the Cochrane literature search was last updated) but considered ineligible for the Cochrane review (four of which were placebo controlled), three trials published before June 2015 that were not identified in the Cochrane search but would likely have been eligible, and 21 trials published since June 2015. Yet, discrepancies in results between the two systematic reviews were not solely attributable to study inclusion criteria. For example, for the effect of vitamin D on pre-eclampsia, De-Regil and colleagues reported a pooled risk ratio of
0.52 (95\% confidence interval 0.25 to 1.05 ) based on two trials that we included in our review, but neither of which met our minimum criteria for case definitions and methods of ascertainment: one trial only mentioned the exclusion of a participant for "severe pre-eclampsia," 38 and the other reported frequencies of pre-eclampsia but did not mention any screening or diagnostic procedures. ${ }^{39}$ De-Regil and colleagues also reported that vitamin $\mathrm{D}$ reduced the risk of preterm birth based on thee trials, ${ }^{6}$ but the current meta-analysis based on 15 comparisons produced reasonably strong evidence of a null effect. These discrepancies highlight well known concerns about the inherent methodological pitfalls of meta-analysis ${ }^{40}$ and the inconsistencies and conflicting results across many published systematic reviews. ${ }^{41} 42$ Although we preregistered this review, followed standard guidelines, and carefully considered heterogeneity and missing data, the selection of studies and outcome data and our choices of analytical approaches were unavoidably subjective and thus prone to bias. We contacted authors to clarify some outcome data to enable its inclusion in the meta-analysis but did not expect authors to do time consuming new analyses (such as incidence rates for acute respiratory infection). A narrative review of findings of five vitamin $\mathrm{D}$ trials that were considered ineligible did not materially alter any of the inferences from the meta-analyses, suggesting that our conclusions were not biased by overly stringent inclusion criteria. It is possible that more robust findings would result from individual participant data meta-analyses, but this would not rectify the problem of low quality trial methods and scant data related to key clinical outcomes (such as pre-eclampsia).

The most important limitation of this systematic review was that it was a snapshot amid a rapidly changing evidence base. Although we intend to update this review regularly (the platform for future real time dissemination is still to be determined), we project limited aggregate gains from the 35 mostly small trials of prenatal vitamin D supplementation trials that are registered as completed, ongoing, or planned. Many of these randomised controlled trials will probably never be published ${ }^{43}$; and, among those that are, it is uncertain whether outcomes will be completely or faithfully reported. ${ }^{44}$ Some current trials will provide clinically relevant information about effects on fetal/infant growth and maternal HIV progression, but any positive findings will need to be confirmed to reliably form the basis for global policy or clinical recommendations. Ultimately, large multicentre dose ranging studies are required to precisely quantify effects of prenatal interventions on important clinical outcomes including hypertensive diseases of pregnancy, gestational diabetes, maternal and neonatal morbidity, and infant developmental outcomes. In the meantime, we endorse the notion that whenever feasible, investigators should report all clinically important outcomes, even in small trials that are individually underpowered to detect effects on such outcomes. ${ }^{45}$ 
John Ioannidis recently remarked that "systematic reviews may sometimes be most helpful if, instead of focusing on the summary of the evidence, highlight the biases that are involved and what needs to be done to remedy the state-of-the-evidence in the given field." ${ }^{46}$ We conclude that there is currently insufficient evidence to guide recommendations for prenatal vitamin D supplementation. Cautious projections for the next decade suggest that we will eventually know more about vitamin D in pregnancy than we do now, but in the absence of a coordinated effort and funding to conduct large new trials, some of the most critical questions about the effectiveness of prenatal vitamin D supplementation will probably remain unanswered in the foreseeable future.

We thank the following authors who generously provided summary statistics or clarifications about their published findings: Sima Hashemipour (Qazvin University of Medical Science, Qazvin, Iran); Aanchal Sablok (Vardhman Mahavir Medical College, New Dehli, India); Bruce Hollis, Carol Wagner, and Myla Ebeling (Medical University of South Carolina, Charleston, US); Christine Rodda (University of Melbourne, Victoria, Australia) and Amanda Vincent (Monash University, Victoria, Australia); Nazli Hossain (Dow University of Health Sciences, Karachi, Pakistan); Mojgan Mirghafourvand (Tabriz University of Medical Sciences, Tabriz, Iran); Adekunle Dawodu (Cincinnati Children's Hospital Medical Center, Cincinnati, US); Cyrus Cooper and Rebecca Moon (University of Southampton, Southampton, UK); Cameron Grant (University of Auckland, Auckland, New Zealand).

Contributors: DER and EM designed the study and wrote the protocol. ML and EM selected articles and performed the assessment of bias. $\mathrm{HQ}$ and JW identified registered trials. ML, EM, HQ, JW, and EP extracted data. ML performed the statistical analysis. DER supervised study activities and wrote the first draft of the manuscript. All authors critically reviewed and revised the manuscript and approved the final version for publication. DER is guarantor.

Funding: This work was supported by the Hospital for Sick Children through support to DER.

Competing interests: All authors have completed the ICMJE uniform disclosure form at www.icmje.org/coi_disclosure.pdf and declare: no support from any organisation for the submitted work; no financial relationships with any organisations that might have an interest in the submitted work in the previous three years; no other relationships or activities that could appear to have influenced the submitted work. Ethical approval: Not required.

Data sharing: The dataset is available from the lead author on request. Transparency: The lead author affirms that the manuscript is an honest, accurate, and transparent account of the study being reported; that no important aspects of the study have been omitted; and that any discrepancies from the study as planned (and, if relevant, registered) have been explained.

This is an Open Access article distributed in accordance with the Creative Commons Attribution Non Commercial (CC BY-NC 4.0) license, which permits others to distribute, remix, adapt, build upon this work non-commercially, and license their derivative works on different terms, provided the original work is properly cited and the use is noncommercial. See: http://creativecommons.org/licenses/by-nc/4.0/.

1 Ross AC, Taylor CL, Yaktine AL, Del Valle HB. Committee to Review Dietary Reference Intakes for Vitamin D and Calcium, Institute of Medicine. Dietary Reference Intakes for Calcium and Vitamin D. The National Academies Press, 2010.

2 Zhang R, Li B, Gao X. Serum 25-hydroxyvitamin D and the risk of cardiovascular disease: dose-response meta-analysis of prospective studies. Am J Clin Nutr 2017;105:810-9. doi:10.3945/ ajcn.116.140392

3 Keum N, Giovannucci E. Vitamin D supplements and cancer incidence and mortality: a meta-analysis. Br / Cancer 2014:111:976-80. doi:10.1038/bjc.2014.294

4 Martineau AR, Jolliffe DA, Hooper RL. Vitamin D supplementation to prevent acute respiratory tract infections: systematic review and meta-analysis of individual participant data. BMJ 2017;356:i6583. doi:10.1136/bmj.i6583

5 Martineau AR, Cates CJ, Urashima M. Vitamin D for the management of asthma. Cochrane Database Syst Rev 2016:9:CD011511.
6 De-Regil LM, Palacios C, Lombardo LK, Peña-Rosas JP. Vitamin D supplementation for women during pregnancy. Cochrane Database Syst Rev 2016;1:CD008873.

7 Palacios C, Gonzalez L. Is vitamin D deficiency a major global public health problem?/ Steroid Biochem Mol Biol 2014;144(Pt A):138-45. doi:10.1016/j.jsbmb.2013.11.003

8 Seamans KM, Cashman KD. Existing and potentially novel functional markers of vitamin D status: a systematic review. Am J Clin Nutr 2009;89:1997S-2008S. doi:10.3945/ajen.2009.27230D

9 Pérez-López FR, Pasupuleti V, Mezones-Holguin E. Effect of vitamin D supplementation during pregnancy on maternal and neonatal outcomes: a systematic review and meta-analysis of randomized controlled trials. Fertil Steril 2015;103:1278-88.e4. doi:10.1016 i.fertnstert.2015.02.019

10 Thorne-Lyman A, Fawzi WW. Vitamin D during pregnancy and maternal, neonatal and infant health outcomes: a systematic review and meta-analysis. Paediatr Perinat Epidemiol 2012;26(Suppl 1): 75-90. doi:10.1111/j.1365-3016.2012.01283.x

11 Harvey NC, Holroyd C, Ntani G. Vitamin D supplementation in pregnancy: a systematic review. Health Technol Assess 2014;18: 1-190. doi:10.3310/hta18450

12 World Health Organization. Recommendations on Antenatal Care for a Positive Pregnancy Experience. WHO, 2016. http://www.who.int/ reproductivehealth/publications/maternal_perinatal_health/ anc-positive-pregnancy-experience/en/.

13 Moher D, Liberati A, TetzlaffJ, Altman DGPRISMA Group. Preferred reporting items for systematic reviews and meta-analyses: the PRISMA statement. Ann Intern Med 2009;151:264-9, W64. doi:10.7326/0003-4819-151-4-200908180-00135

14 Higgins JPT, Green S. Cochrane Handbook for Systematic Reviews of Interventions Version 5.1.0 [updated March 2011]: The Cochrane Collaboration; 2011. http://www.handbook.cochrane.org/.

15 Higgins JP, Altman DG, Gøtzsche PCCochrane Bias Methods GroupCochrane Statistical Methods Group. The Cochrane Collaboration's tool for assessing risk of bias in randomised trials. BMJ 2011;343:d5928. doi:10.1136/bmj.d5928

16 Higgins JP, Thompson SG. Quantifying heterogeneity in a metaanalysis. Stat Med 2002;21:1539-58. doi:10.1002/sim.1186

17 Gagnier JJ, Moher D, Boon H, Beyene J, Bombardier C. Investigating clinical heterogeneity in systematic reviews: a methodologic review of guidance in the literature. BMC Med Res Methodol 2012;12:111. doi:10.1186/1471-2288-12-111

18 Friedrich JO, Adhikari NK, Beyene J. Inclusion of zero total event trials in meta-analyses maintains analytic consistency and incorporates all available data. BMC Med Res Methodol 2007;7:5. doi:10.1186/1471-2288-7-5

19 Higgins JPT, Green S. Cochrane Collaboration. Cochrane handbook for systematic reviews of interventions. Wiley-Blackwell, 2008.

20 Akl EA, Kahale LA, Agoritsas T. Handling trial participants with missing outcome data when conducting a meta-analysis: a systematic survey of proposed approaches. Syst Rev 2015;4:98. doi:10.1186/ s13643-015-0083-6

21 Mavridis D, Chaimani A, Efthimiou O, Leucht S, Salanti G. Addressing missing outcome data in meta-analysis. Evid Based Ment Health 2014:17:85-9 doi:10.1136/eb-2014-101900

22 Aghajafari F, Nagulesapillai T, Ronksley PE, Tough SC, O’Beirne M, Rabi DM. Association between maternal serum 25-hydroxyvitamin D level and pregnancy and neonatal outcomes: systematic review and meta-analysis of observational studies. BMJ 2013;346:f1169. doi:10.1136/bmj.f1169

23 Eckhardt CL, Gernand $A D$, Roth DE, Bodnar LM Maternal vitamin D status and infant anthropometry in a US multi-centre cohort study. Ann Hum Biol 2015;42:217-24. doi:10.3109/03014460.2014. 954616

24 Finch SL, Rauch F, Weiler HA. Postnatal vitamin D supplementation following maternal dietary vitamin D deficiency does not affect bone mass in weanling guinea pigs. J Nutr 2010;140:1574-81. doi:10.3945/in.109.113977

25 Rummens K, van Bree R, Van Herck E. Vitamin D deficiency in guinea pigs: exacerbation of bone phenotype during pregnancy and disturbed fetal mineralization, with recovery by $1,25(\mathrm{OH}) 2 \mathrm{D} 3$ infusion or dietary calcium-phosphate supplementation. Calcif Tissue Int 2002;71:364-75. doi:10.1007/s00223-001-2125-4

26 Bell TD, Demay MB, Burnett-Bowie SA. The biology and pathology of vitamin D control in bone. / Cell Biochem 2010;111:7-13. doi:10.1002/jcb.22661

27 Roth DE, Gernand AD, Morris SK. Maternal vitamin D supplementation during pregnancy and lactation to promote infant growth in Dhaka, Bangladesh (MDIG trial): study protocol for a randomized controlled trial. Trials 2015;16:300. doi:10.1186/s13063-015-0825-8

28 Cooper C, Harvey NC, Bishop NJMAVIDOS Study Group. Maternal gestational vitamin D supplementation and offspring bone health (MAVIDOS): a multicentre, double-blind, randomised placebocontrolled trial. Lancet Diabetes Endocrinol 2016;4:393-402. doi:10.1016/S2213-8587(16)00044-9 
29 Harvey NC, Javaid K, Bishop NMAVIDOS Study Group. MAVIDOS Maternal Vitamin D Osteoporosis Study: study protocol for a randomized controlled trial. Trials 2012;13:13. doi:10.1186/ 1745-6215-13-13

30 Munns CF, Shaw N, Kiely M. Global Consensus Recommendations on Prevention and Management of Nutritional Rickets. / Clin Endocrinol Metab 2016;101:394-415. doi:10.1210/jc.2015-2175

31 Harrington J, Perumal N, Al Mahmud A, Baqui A, Roth DE. Vitamin D and fetal-neonatal calcium homeostasis: findings from a randomized controlled trial of high-dose antenatal vitamin D supplementation. Pediatr Res 2014;76:302-9. doi:10.1038/pr.2014.83

32 Vahdaninia M, Mackenzie H, Helps S, Dean T. Prenatal Intake of Vitamins and Allergic Outcomes in the Offspring: A Systematic Review and Meta-Analysis. J Allergy Clin Immunol Pract 2017;5:771-778.e5. doi:10.1016/j.jaip.2016.09.024

33 Litonjua AA, Carey VJ, Laranjo N. Effect of Prenatal Supplementation With Vitamin D on Asthma or Recurrent Wheezing in Offspring by Age 3 Years: The VDAART Randomized Clinical Trial. JAMA 2016;315: 362-70. doi:10.1001/jama.2015.18589

34 Chawes BL, Bønnelykke K, Stokholm J. Effect of vitamin d3 supplementation during pregnancy on risk of persistent wheeze in the offspring: A randomized clinical trial. JAMA 2016;315:353-61. doi:10.1001/jama.2015.18318

35 Lappe JM, Heaney RP. Why randomized controlled trials of calcium and vitamin D sometimes fail. Dermatoendocrinol 2012;4:95-100. doi:10.4161/derm.19833

36 Aloia JF, Patel M, Dimaano R. Vitamin D intake to attain a desired serum 25-hydroxyvitamin D concentration. Am J Clin Nutr 2008;87:1952-8.

37 Roth DE, Al Mahmud A, Raqib R, Akhtar E, Black RE, Baqui AH. Pharmacokinetics of high-dose weekly oral vitamin D3 supplementation during the third trimester of pregnancy in Dhaka, Bangladesh. Nutrients 2013:5:788-810. doi:10.3390/nu5030788

38 Asemi Z, Samimi M, Tabassi Z, Shakeri H, Esmaillzadeh A. Vitamin D supplementation affects serum high-sensitivity C-reactive protein, insulin resistance, and biomarkers of oxidative stress in pregnant women. J Nutr 2013;143:1432-8. doi:10.3945/jn.113.177550

39 Sablok A, Batra A, Thariani K. Supplementation of vitamin D in pregnancy and its correlation with feto-maternal outcome. Clin Endocrinol (Oxf) 2015;83:536-41. doi:10.1111/cen.12751

40 Eysenck HJ. Meta-analysis and its problems. BMJ 1994;309:789-92 doi:10.1136/bmj.309.6957.789

41 Alexander DD, Weed DL. On the need for improved methodologic quality of published reviews. Am J Clin Nutr 2016;103:683-4. doi:10.3945/ajcn.115.130005

42 Ioannidis JP. The Mass Production of Redundant, Misleading, and Conflicted Systematic Reviews and Meta-analyses. Milbank Q 2016;94:485-514. doi:10.1111/1468-0009.12210
43 Chen R, Desai NR, Ross JS. Publication and reporting of clinical tria results: cross sectional analysis across academic medical centers. BM/ 2016;352:i637. doi:10.1136/bmj.i637

44 Jones CW, Keil LG, Holland WC, Caughey MC, Platts-Mills TF. Comparison of registered and published outcomes in randomized controlled trials: a systematic review. BMC Med 2015;13:282 doi:10.1186/s12916-015-0520-3

45 Ioannidis JP, Horbar JD, Ovelman CM. Completeness of main outcomes across randomized trials in entire discipline: survey of chronic lung disease outcomes in preterm infants. BMJ 2015;350:h72. doi:10.1136/bmj.h72

46 Ioannidis JPA. Retraction Watch. http://retractionwatch. com/2016/03/16/evidence-based-medicine-has-been-hijacked-aconfession-from-john-ioannidis/2016

Appendix 1: Selected recent recommendations for vitamin D intake or supplementation in pregnancy Appendix 2: Search strategies for electronic databases

Appendix 3: Outcome variable case definitions and methods of ascertainment criteria

Appendix 4: Search strategy for clinical trial registries

Appendix 5: Studies included in systematic review and eligible for inclusion in meta-analyses

Appendix 6: Contributions of prenatal vitamin D trials to meta-analyses

Appendix 7: Unpublished outcome data and clarifications of published findings

Appendix 8: Prenatal prospective controlled vitamin D supplementation trials ineligible for inclusion in meta-analyses

Appendix 9: Risk of bias for trials eligible for inclusion

Appendix 10: Sensitivity analyses and sub-group meta-analyses and meta-regression

Appendix 11: Future projections 